\title{
La comunicación visual de los productos a través del packaging. Un estudio de caso sobre la identidad visual de las botellas de leche
}

\author{
Produktuen komunikazio bisuala packaging-aren \\ bitartez. Esne-botilen identitate bisualari \\ buruzko kasu-azterketa
}

\section{Visual communication of products through packaging. A case study on the visual identity of milk bottles}

\author{
Jordi Colet Ruz ${ }^{1}$
}

\section{zer}

Vol. 21 - Núm. 40

ISSN: $1137-1102$

e-ISSN: $1989-631 X$

DOI: $10.1387 /$ zer. 15513

pp. $195-217$

2016

Recibido el 20 de enero de 2016, aceptado el 11 de abril de 2016.

\section{Resumen}

Los envases se han convertido en una pieza significativa del ecosistema visual que tiene un gran potencial comunicativo. Este potencial debe incluir la capacidad de transmitir la identidad tipológica del producto en un escenario muy competitivo. Esta investigación aborda la influencia del packaging en la identificación tipológica de los productos de alta frecuencia de compra. El objeto de estudio se ha focalizado en el código visual que regula el diseño de los envases, con el objeto de facilitar el reconocimiento tipológico del producto. Aquí se presenta un estudio de caso sobre el código visual en las botellas de leche.

Palabras-clave: Comunicación, diseño, marketing, percepción, publicidad.

\section{Laburpena}

Ontziak ekosistema bisualaren pieza esanguratsua bihurtu dira, eta komunikatzeko ahalmen handia dute. Ahalmen horrek produktuaren identitate tipologikoa transmititzeko gaitasuna izan behar du kontuan, agertokia oso lehiakorra baita. Ikerketa honek packaging-ak maiz erosten diren produktuen identifikazio tipologikoan duen eragina jorratzen du. Azterketak ontzien diseinua arautzen duen kode bisuala du ardatz, produktuaren azterketa tipologikoa errazteko helburuarekin. Hemen, esne-botilen kode bisualari buruzko kasu-azterketa bat aurkezten da.

Gako-hitzak: Komunikazioa, diseinua, marketina, pertzepzioa, publizitatea. 


\begin{abstract}
Packaging has become a significant part of the visual ecosystem which has a great potential for communication. This potential should include the ability to transmit the typological identity of product in a highly competitive scenario. This research approaches the influence of packaging on the typological identification of fast-moving consumer goods. The object of study has focused on the visual code that regulates packaging in order to facilitate the typological product recognition. This paper presents a case study on the visual code of milk bottles.
\end{abstract}

Key-words: Communication, design, marketing, perception, advertising. 


\section{Introducción}

La función de los envases se basa en la ineludible necesidad de contener, conservar y transportar productos. Sin embargo, dichos artefactos han experimentado una profunda transformación que los ha convertido en un instrumento al servicio del marketing. A través del packaging se logra articular la acción publicitaria de los productos en el punto de venta. La influencia comunicativa de los envases sobre el comportamiento de compra es significativa y su diseño gráfico puede representar una metáfora visual de nuestra sociedad contemporánea.

La construcción de la identidad visual de los productos tiene el difícil reto de facilitar la identificación inmediata del producto y, al mismo tiempo, transmitir los valores que conforman el posicionamiento publicitario de la marca. En este sentido, el diseño de los envases es la manifestación más elocuente de este reto.

La presente investigación expone los resultados de un proyecto experimental implementado para contrastar las hipótesis de trabajo expuestas por el autor en el marco de una tesis doctoral que aborda el estudio de la identidad visual de los envases desde la perspectiva del mensaje gráfico publicitario. En este sentido, el trabajo pretende ser una aportación al conocimiento de la denominada alfabetidad visual la cual, de acuerdo con Dondis (2004: 208), "significa una mayor inteligencia visual".

En definitiva, conocer la sintaxis visual en relación al diseño estructural y gráfico de los envases ha sido el principal propósito del trabajo; particularmente, la que afecta a los envases concebidos para los productos de alta frecuencia de compra (fast-moving consumer goods). Desde esta perspectiva, el resultado deberá permitir la formulación de mensajes más eficientes y, en consecuencia, mejorar el rendimiento comunicativo de los envases.

\section{Estado de la cuestión}

El vínculo entre la inteligencia humana y la realidad física -de carácter extracognitivo- se establece a través de la facultad perceptiva multisensorial de los seres humanos. Por esta razón, la recepción sensorial se erige como una causa fundamental que incide en la formación de la inteligencia humana. Todas las cosas que vemos, escuchamos, olemos, degustamos y tocamos delimitan nuestros aprendizajes, experiencias, sensaciones y pensamientos; son los estímulos a partir de los cuales se construye la propia inteligencia y una determinada -y necesariamente limitada- concepción del mundo exterior.

Inmersos en un entorno comercial afectado por una alta saturación de estímulos sensoriales, la identidad visual de los envases deviene un factor relevante en este ecosistema que incorpora un gran potencial comunicativo. De hecho, el punto de venta los ha transformado en unos auténticos artefactos publicitarios de gran importancia estratégica para las marcas. El desarrollo de este potencial queda sintetizado por la capacidad de transmitir la identidad tipológica del producto y de la marca en un momento decisivo: el acto de compra.

El problema de conocimiento que plantea esta investigación versa sobre cómo la identidad visual de los envases permite la identificación tipológica del producto y, al mismo tiempo, de qué manera este hecho puede provocar una afectación sobre la 
preferencia del receptor. Es decir, el objetivo es conocer cómo se formula el mensaje visual a fin de potenciar la comunicación publicitaria del envase en el punto de venta.

En base a lo expuesto, el objeto de estudio se ha centrado en la existencia de un código visual y en los efectos positivos que se derivan de su aplicación sobre el comportamiento de los sujetos. En otras palabras, la finalidad de la investigación es contrastar si la identidad visual de los envases se encuentra regida, en mayor o menor medida, por un código visual. Por este motivo, la observación del receptor, expuesto a una determinada formulación visual, es un requerimiento básico para el estudio de su comportamiento de compra. Como apunta Rom (2002: 152), "la posibilidad de establecer códigos visuales para facilitar el acto de comunicación y aumentar la eficacia de los mensajes hace necesario el estudio del imaginario iconográfico de los públicos del diseño". Precisamente, esto es lo que aquí se ha estudiado de forma experimental; cómo se establecen premeditadamente unas formulaciones visuales en torno a un caso particular: las botellas de leche. En definitiva, el trabajo pretende concretar si determinados prototipos visuales son portadores de un código tipológico que determina la identidad estructural y gráfica de los envases de leche.

Desde una perspectiva científica, abordar el estudio del packaging supone un reto para el avance del conocimiento en diseño gráfico, para convertir los envases en artefactos más eficaces y eficientes a nivel comunicativo. En palabras de Bunge (1989: 45), "la investigación científica es, dicho brevemente, la búsqueda de estructuras". Es decir, la ciencia se fundamenta en la búsqueda de categorías e interrelaciones para comprender y transformar -cuando esto sea posible- el funcionamiento de las cosas.

La teoría general de los sistemas formulada por Ludwing von Bertalanffy (1981: $45)$ se sustenta en esta idea inicial. Bertalanffy proporcionó un nuevo enfoque a la investigación científica sobre la base que todo objeto de estudio forma parte de una realidad más global. Una realidad determinada por los hechos y explicada a partir de la investigación sobre los componentes que la integran y las relaciones que se establecen. Desde este punto de vista, no es casual que este autor justifique "la aparición, en todos los campos de la ciencia, de nociones como las de totalidad, holismo, organismo, Gestalt, etc., que vienen a significar todas que, en última instancia, debemos pensar en términos de sistemas de elementos en interacción mutua."

Los primeros paradigmas comunicativos se inspiran en este principio sistémico. La investigación de la comunicación humana como disciplina científica arranca a mediados del siglo XX cuando se presentan los modelos comunicativos de Harold D. Lasswell y Claude E. Shannon, entre otros (Saperas, 1985). Estos primeros modelos se originan en el marco de la Mass Communication Research con la finalidad de explicar los principios que regulan la comunicación y, al mismo tiempo, establecer unas incipientes bases teóricas sobre el tema. De hecho, el interés de la investigación científica en este ámbito se centraba en analizar, fundamentalmente, los efectos de los medios de comunicación de masas; es decir, la capacidad de influencia de los medios en la opinión pública.

Estos primeros modelos comunicativos han quedado como referentes históricos. En la actualidad, la comunicación en general -y muy especialmente, la publicitaria- ha experimentado numerosos cambios para adaptarse a las nuevas necesidades y exigencias de una sociedad moderna, más compleja y diversa, sobresaturada de estímulos, en la cual la creatividad tiene que hacer un gran esfuerzo de conceptualización de los mensajes. 
Una de estas formas de expresión publicitaria se ha manifestado a través del packaging. Las actuales exigencias y hábitos de consumo -los nuevos formatos de producto, la usabilidad de los envases o el impacto medioambiental que generan, entre otros- han incentivado su crecimiento económico y, también, su desarrollo técnico y comunicativo. En este contexto, la función publicitaria del envase ha sido decisiva. Los envases han devenido algo más que unos artefactos donde contener, conservar y transportar unos determinados productos; son, sobretodo, la expresión visual de un mensaje. Desde este punto de vista, se erigen como una auténtica y valiosa herramienta de marketing en un mercado de consumo donde las características, la calidad y el precio de los productos pueden resultar muy similares (Veryzer, 1995). En suma, los envases permiten comunicar y generar identidad de marca, especialmente en un mercado de fuerte competencia (Connolly, 1996; Rettie, 2000). Como bien dice Bassat (1994: 35), no podemos olvidar la función publicitaria del envase dado que "es el último interlocutor de nuestro cliente, en él delegamos el cierre de la venta, y para ello debemos dotarlo de toda la información, de toda la capacidad de persuasión y de toda la personalidad diferencial que sea posible". Ante productos similares en calidad y precio, la comunicación publicitaria a través del envase es crucial. Por este motivo, el packaging es un factor determinante que afecta el comportamiento de compra (Veryzer, 1995).

En el marco de esta concepción sistémica del proceso comunicativo, una primera reflexión en torno al packaging permite plantear una modelización de dicho proceso. A pesar de que, en palabras de Arnheim (2005: 73), "los constructos teóricos nunca pretenden otra cosa que dar una versión aproximada de las complejidades de la realidad", se trata de un punto de partida útil y necesario. Su finalidad es ofrecer una perspectiva general del proceso para situar adecuadamente el objeto de estudio y, a la vez, representar gráficamente su carácter sistémico.

La referida propuesta de modelización (Figura 1) contempla cuatro grandes dimensiones interdependientes las cuales corresponden a las etapas de concepción, producción, difusión y recepción del mensaje. No obstante, en esta investigación sólo se desarrolla la dimensión comunicativa del pack desde la perspectiva del mensaje gráfico publicitario.

En términos generales, el origen de la actividad comunicativa parte de un emisor; de una persona física o jurídica que tiene la necesidad o el propósito de transmitir un mensaje. Naturalmente, cuando la comunicación tiene carácter comercial, el objetivo es persuadir para provocar un determinado comportamiento de compra.

Dicha modelización considera la dimensión comunicativa como aquella parte del proceso centrada fundamentalmente en la construcción formal del mensaje. Por tanto, su importancia estratégica reside, por un lado, en aquello que se quiere decir sobre el producto pero, por el otro, en cómo se pretende abordar a través del diseño estructural y gráfico de los envases.

De acuerdo con lo dicho, el desencadenante del proceso comunicativo tiene relación con una fuente de información representada por el anunciante o el cliente. Esta fuente de información es la responsable de planificar una determinada estrategia de marketing y, también, de elaborar un briefing a partir del cual el emisor -léase diseñador o codificador- dispondrá de los requerimientos básicos para formular y defender las propuestas de mensaje visual. Sea una agencia de publicidad o estudio 
de comunicación, el emisor siempre parte de un conjunto de datos sin los cuales el proyecto va a resultar inviable e infructuoso.

Cuando el anunciante decide la promoción de un producto, la agencia de publicidad y el equipo de diseño se erigen como el emisor en tanto que reciben el encargo de concebir el mensaje a partir de unos objetivos. Conceptualmente, el emisor tiene la responsabilidad de definir la identidad visual del envase, desde la estrategia comunicativa hasta la concepción estructural y gráfica del artefacto. En este mismo sentido se posiciona Dondis $(2004,33)$ cuando para referirse al proceso de composición visual dice que "en esta etapa vital del proceso creativo, es donde el comunicador visual ejerce el control más fuerte sobre su trabajo y donde tiene la mayor oportunidad para expresar el estado de ánimo total que se quiere transmita la obra". De hecho, la función primordial de este equipo de trabajo multidisciplinar -en el cual los creativos marcan una estrategia comunicativa y los diseñadores plantean probables formulaciones visuales- es la definición conceptual y formal del mensaje (Figura 2).

Figura 1: Modelización del proceso de comunicación publicitaria a través de los envases.

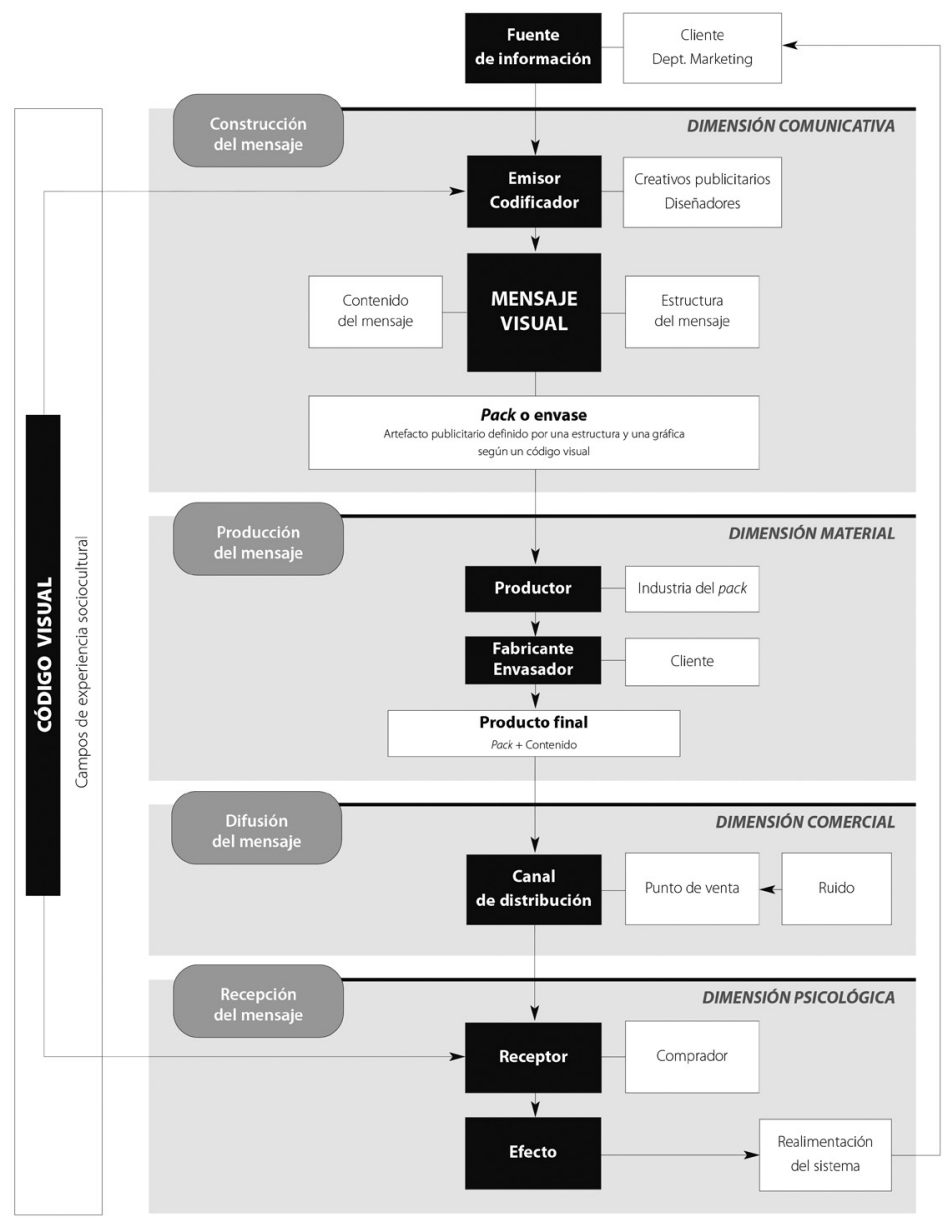

Fuente: Elaboración propia. 
Figura 2: Proceso de construcción formal del mensaje publicitario en los envases.

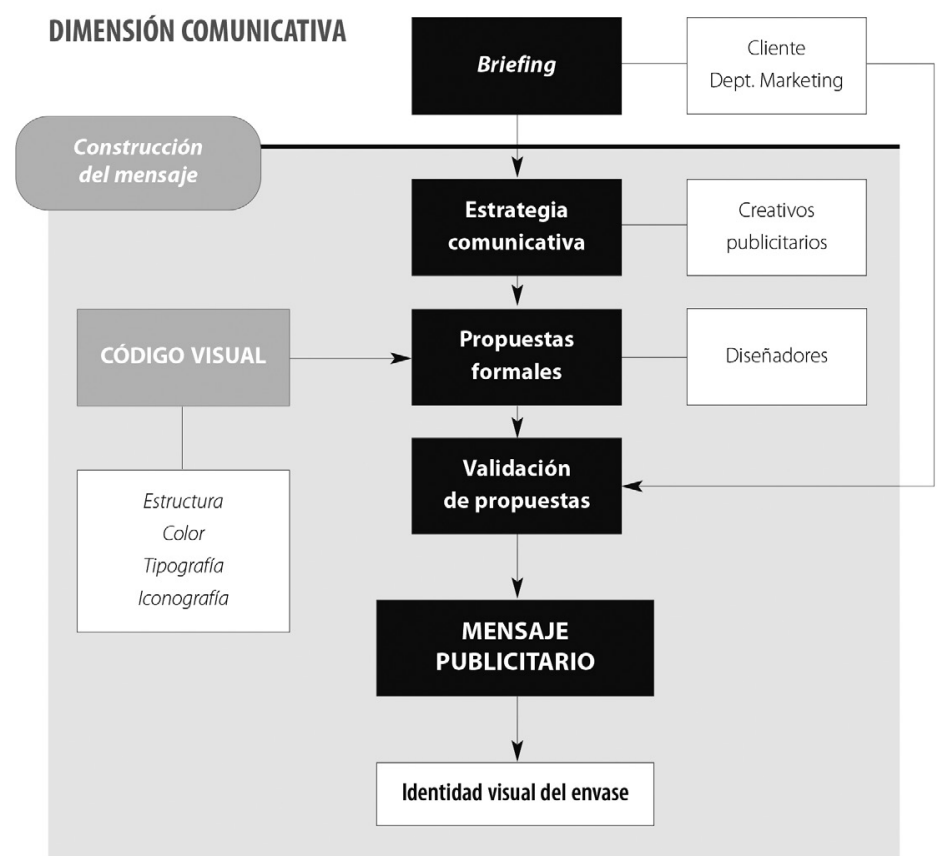

Fuente: Elaboración propia.

Atendiendo a su dimensión comunicativa, el envase no puede ser considerado un simple contenedor. Su diseño estructural y gráfico permite la representación de la propia identidad visual de los productos. De hecho, transforma el envase en portador de un mensaje publicitario que debe persuadir al comprador. Como afirma Vidales (2003: 21), "el envase es considerado como un objeto-mensaje que participa de un proceso de comunicación". Aunque el aspecto exterior del pack no sea considerado stricto sensu el producto, las propiedades estructurales y gráficas del artefactocontenedor devienen una parte indisociable del mismo. Desde esta perspectiva, los envases también se conciben como objeto de consumo dado que participan activamente del proceso de compra, particularmente en algunas tipologías de producto. En muchos casos, las propiedades funcionales, nutritivas o terapéuticas del contenido son sólo una condición necesaria aunque no suficiente para lograr la preferencia del consumidor. Según Viñolas (2005: 323), “en la actualidad y salvo raras excepciones, la esencia de los envases no son los productos sino la imagen, las asociaciones y metaprestaciones, de modo que éstas se han convertido en el soporte de las marcas y en símbolo de su prestigio". Por esta razón, los envases sobrepasan las propiedades del contenido y devienen parte significativa del producto que se pretende vender.

Desde un planteamiento semiótico, este conjunto de propiedades estructurales y gráficas de los envases son signos visuales, elementos de significación, creados y organizados según una determinada convención cultural. Como expone Eco (1972), se dan códigos arquitectónicos que indican qué forma tienen las construcciones -o los 
objetos que forman parte de nuestro entorno- a través de las cuales se puede reconocer la identidad de las mismas. En este sentido, Vidales $(2003,21)$ afirma que "como objetos semióticos, los envases y embalajes son soportes de información, vehículos de mensajes, portadores de significados".

Asimismo, el lenguaje visual de los productos es un hecho social y cultural; un patrimonio colectivo determinado -según la sociología de la comunicación- por los campos de experiencia. De acuerdo con Saperas $(1985,83)$ dicho concepto se utiliza para definir "el conjunto de conocimientos de naturaleza cultural o aprehendidos a lo largo de la actividad cotidiana que permiten a cada individuo determinar su conducta en cada ocasión según sus propios conocimientos". Otros autores como DeFleur (1982) se refieren a las construcciones socioculturales de la realidad. En este sentido, la presencia de un código visual en el packaging es una expresión particular de la cultura visual de nuestra sociedad; una parte de la memoria colectiva que surge como consecuencia de unos espacios de relación comercial, social y cultural.

La apariencia de los envases, su aspecto visual, es un factor importante que influye al comprador durante el proceso de selección de un producto en el punto de venta (Bloch, 1995; Garber, 1995; Veryzer, 1995; Sierra, 2001; Martínez Bouza, 2010); en particular, si se considera que más de la mitad de las decisiones de compra se adoptan habitualmente en el mismo punto de venta o bien no estaban previamente planificadas (Philips, 1993; Connolly, 1996).

Para abordar la cuestión sobre la identidad visual de los envases se ha adoptado el concepto de Estado Estético propuesto por Max Bense y recuperado por Daniel Tena (1998). En este sentido, cualquier artefacto es portador de un determinado Estado Estético que se origina mediante el concurso de diversos elementos visuales $y$ gráficos, y con la finalidad de suscitar la preferencia del receptor. Desde la perspectiva del packaging, el Estado Estético trata sobre una formulación visual que configura la identidad de los productos y que, para ser debidamente investigada, debe ser observada a través de la percepción humana. En este caso particular, se pretende su aplicación al estudio de la identidad de los productos de alta frecuencia de compra (fast-moving consumer goods).

Una aproximación a la dimensión comunicativa de estos artefactos permite plantear que la construcción de esta identidad visual pueda estar regulada -en mayor o menor medida- por el concurso de un código específico. Un código instituido a partir de las propiedades estructurales y gráficas del artefacto visual gracias a las cuales se puede identificar el carácter tipológico de los productos y las marcas. ${ }^{2}$ En este caso, la configuración del Estado Estético se articularía por la acción de dicho código.

Considerando que el proceso de selección de un producto tiende-especialmente cuando se trata de actividades rutinarias- hacia la eficiencia de los recursos cognitivos, la presencia de un código tipológico en los envases puede ser un mecanismo útil para agilizar el reconocimiento del producto en el punto de venta. En este sentido, un deficiente uso del código puede inducir a la desorientación o confusión del receptor. Como afirma Eco (1977: 418), "desde el punto de vista semiótico, la ambiguiedad puede definirse como violación de las reglas del código".

\footnotetext{
La Ley de marcas (BOE n ${ }^{\circ} 294,8$ de diciembre de 2001) entiende que las formas tridimensionales, entre las que se incluyen las formas de los envases y los embalajes, pueden considerarse marcas siempre que se atiendan las condiciones que establece la ley.
} 
En relación a la importancia del aspecto visual del producto con respecto a la preferencia del consumidor, Creusen (2005) contempla que una acusada diferencia o similitud con los productos de la misma categoria puede ser una estrategia beneficiosa según la situación. Por esta razón, la baja implicación de los sujetos en procesos de compra asiduos favorece que los productos con un aspecto visual tipificado tengan una mayor aceptación social debido al menor esfuerzo mental requerido (Hoyer, 1984). En estas circunstancias, tipificar el aspecto visual del producto es recomendable (Alba, 1987). En contraposición, cuando el posicionamiento de un producto se fundamenta en el prestigio, la exclusividad o la novedad, es aconsejable que su aspecto se destaque significativamente del resto (Ward, 1988).

Los procesos de aprendizaje, reconocimiento e interpretación de los estímulos sensoriales son la base a partir de la cual el receptor puede articular una determinada respuesta. Pero la percepción no se explica exclusivamente por la presencia de unas sensaciones de naturaleza física canalizadas a través de los sentidos; no es un proceso pasivo de entrada de estímulos sino una actividad centrada en la selección, organización y actualización de los datos que aportan los sentidos (Viglietti, 1975). La sensación tiene carácter reactivo; es simplemente una reacción que se origina por la acción de un estímulo sobre un receptor sensorial que envía un impulso nervioso al cerebro (Schnake, 1990).

Por otra parte, el acto de la percepción es más profundo y complejo. Es un proceso cognitivo que conlleva un filtrado e interpretación de la información. La percepción transforma las sensaciones físicas vehiculadas por los sentidos en significados; es un proceso de semiosis (Costa, 2004).

Las investigaciones realizadas por la Escuela Gestalt sobre la percepción humana han sido un modelo de referencia para la comunicación visual. Los trabajos de esta escuela concluyeron, por un lado, que "el estímulo se percibe como un todo" y, por el otro, que "el individuo posee un mecanismo cognitivo hacia una configuración cognitiva ordenada" (Aaker, 1984: 64). El primero corresponde al principio de la totalidad; la percepción es concebida como una actividad basada en la síntesis de estímulos. El proceso perceptivo se origina a partir de un conjunto de elementos visuales que integran una totalidad, una configuración identificable o gestalt en la cual la percepción de la globalidad es más importante que la de los componentes por separado. El segundo principio defiende que el sistema cognitivo dispone de mecanismos que facilitan la percepción a través de pautas visuales. La ley gestáltica de pregnancia plantea que cuando las sensaciones se organizan para construir formas, el sistema cognitivo las percibe siguiendo el criterio de la simplicidad. La manera más sencilla de alcanzar la simplicidad de un artefacto visual es a través de la reducción y organización de los elementos que lo conforman. Según afirma Maeda (2006: 89), la simplicidad es "sustraer lo que es obvio y añadir lo específico".

Desde el ámbito de la psicología cognitiva también se han realizado aportaciones conceptuales que permiten entender la profundidad del fenómeno perceptivo. Una de las condiciones que intervienen en el funcionamiento del sistema cognitivo es la formación de estructuras mentales; es decir, la organización de la información en categorías para poder clasificar y relacionar el conocimiento en función de determinados criterios taxonómicos (Sierra, 2001). 
La categorización es un concepto que pone de manifiesto la tendencia humana hacia la organización y significación de la experiencia sensorial; una función básica de carácter cognitivo que guía el comportamiento humano. En este sentido, DeFleur (1982: 180) afirma que la percepción es "la actividad mental por la cual el ingreso de datos sensoriales es clasificado dentro de categorías reconocibles de experiencias".

De acuerdo con esto, la creación de categorías por parte del sistema cognitivo obedece a la necesidad de organización del conocimiento para gestionarlo de forma eficaz y eficiente; es decir, permite la identificación tipológica de los productos y las marcas en el punto de venta. Sierra (2001) plantea la función de la apariencia visual de los envase en el proceso de categorización, especialmente cuando el comprador necesita diferenciar los productos, marcas y variedades.

Otros autores como Garber (1995), Bloch (1995) y Veryzer (1995) han destacado la influencia del diseño en la categorización dado que una de las principales funciones de las categorías es diferenciar los productos durante el proceso de compra. En particular, Garber (1995) defiende que el diseño de los envases determina el reconocimiento de los productos a partir de una apariencia visual más o menos prototípica.

Schoormans (1997) recomienda que los envases deberían establecer diferencias evidentes entre los productos, aunque también reconocen que los efectos positivos de la apariencia prototípica pueden desaparecer si no se respetan los códigos visuales establecidos para una determinada categoría. Por otra parte, las aportaciones de Bloch (1995) y Veryzer (1995) sobre esta cuestión indican que la creación de prototipos visuales facilita la categorización de los productos; en concreto, destacan la importancia de la estructura del envase.

Desde la perspectiva del sistema cognitivo, la percepción de disonancia originada por la transgresión de un código tiene importantes implicaciones en el diseño de los envases. Según la teoría de la disonancia cognitiva de Festinger (1975), el comportamiento de los sujetos se rige por el equilibrio entre cognición y acción. Dicha dualidad encuentra el equilibrio necesario cuando entre el pensamiento y el comportamiento se ha establecido una relación de coherencia.

La manifestación de un hecho disonante es algo que produce incomodidad en los sujetos dado que es el resultado de una respuesta no consecuente con lo que se piensa o sabe. "Cuando un consumidor recibe información acerca de cierto producto que no concuerda con lo que él siente hacia el producto, experimenta una incomodidad intelectual o inconsistencia que se denomina disonancia cognitiva" (Schnake, 1990 : 90). Por esta razón, esta incomodidad conlleva habitualmente la justificación del propio comportamiento.

El fenómeno de la disonancia cognitiva aplicado al diseño de los envases ha sido abordado por Julia von Fraunberg ${ }^{3}$. Esta diseñadora ha investigado sobre los efectos del intercambio de identidades gráficas en los envases de algunos productos; en concreto, aquellas que son visualmente más prototípicas para una determinada tipología de producto. Fraunberg plantea una situación de disonancia cognitiva originada por la percepción de la identidad visual de los envases. Un escenario en el cual los sujetos se sienten desconcertados dado que las propiedades estructurales y gráficas del contenedor no se ajustan al contenido real envasado. De hecho, dicha disonan-

3 Su trabajo se encuentra publicado en: CAPELLA, Juli y ÚBEDA, Ramon. Cocos, copias y coincidencias. Barcelona: Editorial Electa, 2003. 
cia está originada por una transgresión del código visual. En suma, el propósito de su investigación era constatar que el proceso de elección de productos envasados -especialmente, cuando son de alta frecuencia de compra- queda condicionado por unos códigos visuales preestablecidos.

\section{Metodología}

En la presente investigación se ha adoptado una metodología experimental. Cada variable se refiere a una propiedad intrínseca del artefacto visual. En concreto, la estructura del envase y dos valores cromáticos específicos han sido las variables independientes a partir de las cuales se determinaron los tratamientos experimentales.

La primera variable del experimento (estructura) corresponde a la forma tridimensional del contenedor; la segunda (color_base) determina el principal valor cromático de su superficie; finalmente, la tercera variable (color_auxiliar) se asocia con otro valor cromático significativo, pero de menor impacto visual.

La estrategia utilizada en esta investigación ha sido un diseño factorial de medidas repetidas (within-subject design). Con este procedimiento estadístico se ha planteado la manipulación simultánea de las tres variables independientes y, consiguientemente, se han podido analizar dos cuestiones de interés: la influencia que cada variable tiene sobre la variable dependiente y, al mismo tiempo, los efectos de la interacción detectados entre las propias variables independientes. Por tanto, se procede a la valoración de los efectos que cada factor provoca en la variable de estudio pero considerando, también, la situación puntual del resto de los factores manipulados en el experimento.

En particular se trata de un diseño (3) x (3) x (3). Es decir, un único grupo se somete a un conjunto de 27 tratamientos experimentales. La primera variable -estructura-tiene 3 niveles; la segunda -color base-dispone de 3 niveles más y, finalmente, la última variable -color auxiliar - también presenta 3 niveles. La exposición del grupo a la totalidad de los tratamientos ha permitido la recogida de los datos necesarios para su posterior análisis estadístico.

El principal propósito de este experimento es contrastar la existencia de un código visual en los envases de leche. En concreto, se quiere saber si la identidad visual de las botellas de leche entera ha quedado establecida por unas especificaciones estructurales y cromáticas determinadas. A continuación se exponen las hipótesis de trabajo que se pretenden validar.

$\mathrm{H}_{1}$ La forma estructural del envase es un componente del código visual.

$\mathrm{H}_{2}$ El valor cromático base del envase es un componente del código visual.

$\mathrm{H}_{3}$ El valor cromático auxiliar del envase es un componente del código visual.

$\mathrm{H}_{4}$ Cuando se combina en un envase el valor cromático base con el valor cromático auxiliar se identifica la tipología del producto.

$\mathrm{H}_{5}$ Cuando se combina la estructura del envase con los valores cromáticos base y auxiliar se identifica la tipología del producto.

Para la realización del experimento se ha utilizado una audiencia cautiva. Es decir, los participantes han formado parte de una muestra de conveniencia integrada por 
un grupo de 45 estudiantes universitarios de la Facultad de Ciencias de la Comunicación de la Universidad Autónoma de Barcelona (UAB) que estaban cursando estudios de Periodismo. En este sentido -y de acuerdo con los planteamientos metodológicos formulados por Igartua (2006) - el uso de las muestras de conveniencia constituidas por estudiantes universitarios es un hecho aceptado y habitual cuando se hacen investigaciones científicas en Comunicación. Un 26\% de la muestra estaba formada por hombres y un $74 \%$ por mujeres, entre 18 y 25 años.

El desarrollo de la prueba experimental se realizó a través de la Plataforma para el Análisis de la Recepción en Comunicación (denominada PARC ${ }^{4}$ ). Se trata de un instrumento al servicio de los investigadores concebido para la preparación, administración y control de los ensayos científicos que se basan en la percepción visual. Esta aplicación ha permitido el montaje del test de recepción el cual presenta, por un lado, una proposición de respuesta cerrada (escala tipo Likert de cinco niveles de respuesta) y, por el otro, unos estímulos visuales premeditadamente manipulados y controlados por el investigador. En esta ocasión, dicho enunciado afirmaba textualmente lo siguiente:

"Esta maqueta corresponde a una botella de leche entera"

\section{Análisis estadístico}

En el contexto de la estadística inferencial, se procede a efectuar una valoración preliminar sobre el funcionamiento del instrumento de medida implementado en este experimento. En este sentido, la medida de la fiabilidad del instrumento se convierte en un indicador básico de análisis que tiene el objetivo de garantizar la calidad de los datos obtenidos. De acuerdo con esto, se ha controlado la capacidad métrica del cuestionario con la aplicación informática SPSS Statistics y a través del coeficiente Alfa de Cronbach.

Según los resultados obtenidos, se comprueba que la fiabilidad del instrumento es muy buena $(0,875)$ dado que el valor obtenido se encuentra entre 0,70 y 0,90 . Por tanto, se puede asegurar que los ítems de la escala miden efectivamente el mismo atributo.

Se ha adoptado un diseño factorial de la varianza con medidas repetidas para poder valorar, por una parte, los efectos principales de las variables independientes sobre la variable dependiente $\mathrm{y}$, por otra, los posibles efectos de interacción entre las variables objeto de estudio. En este sentido, el análisis de los efectos es la base a partir de la cual se podrá contrastar en qué medida las hipótesis formuladas son válidas.

En el análisis estadístico se ha considerado el nivel de significación y la potencia del contraste como criterios básicos de decisión. Asimismo, el procedimiento utilizado para analizar los datos obtenidos ha sido el GLM (General Linear Model).

\subsection{Primera hipótesis}

$\mathrm{H}_{1}$ La forma estructural del envase es un componente del código visual.

\footnotetext{
${ }^{4}$ La información técnica sobre la Plataforma para el Análisis de la Recepción en Comunicación (PARC) ha sido publicada en: LÁZARO, Patricia; TENA, Daniel; BLANCO, Josep Maria; VOCES, Ramon (2007). La recepción, un límite a la creación. Trípodos (número extraordinario), pp. 685-704.
} 
Dado que el nivel de significación (sig.) relativo al factor Estructura es 0,001 -por tanto, inferior a 0,05- se puede rechazar la hipótesis nula y, en consecuencia, concluir que se detectan diferencias significativas entre las estructuras de la botella (Tabla 1). Dicho en términos estadísticos, se dispone de suficientes garantías para no incurrir en un error Tipo I y, por tanto, se puede aceptar la credibilidad de dicha hipótesis.

Asimismo es importante considerar la excelente potencia estadística del contraste $(0,968)$ la cual, como puede comprobarse, es bastante superior a 0,80. Por tanto, la probabilidad de cometer un error Tipo II es muy baja.

Por otra parte, las medias marginales estimadas del factor Estructura (Tabla 2) relativas a las maquetas A, B y C (Figura 3) indican que la media más alta corresponde a la maqueta $B(2,291)$.

Tabla 1. Valores estadísticos de significación y potencia de los contrastes relativos al factor Estructura.

\begin{tabular}{|l|c|c|c|c|c|c|c|}
\hline \multicolumn{7}{|c|}{ ESTRUCTURA } \\
\hline $\begin{array}{l}\text { Contrastes } \\
\text { multivariados }\end{array}$ & Valor & F & $\begin{array}{c}\text { GI de la } \\
\text { hipótesis }\end{array}$ & $\begin{array}{c}\text { GI del } \\
\text { error }\end{array}$ & Sig. & $\begin{array}{c}\text { Coeficiente } \\
\mathbf{r}^{2}\end{array}$ & $\begin{array}{c}\text { Potencia } \\
\text { observada }\end{array}$ \\
\hline Traza de Pillai &, 316 & 9,244 & 2,000 & 40,000 &, 001 &, 316 &, 968 \\
\hline $\begin{array}{l}\text { Lambda } \\
\text { de Wilks }\end{array}$ &, 684 & 9,244 & 2,000 & 40,000 &, 001 &, 316 &, 968 \\
\hline $\begin{array}{l}\text { Traza } \\
\text { de Hotelling }\end{array}$ &, 462 & 9,244 & 2,000 & 40,000 &, 001 &, 316 &, 968 \\
\hline $\begin{array}{l}\text { Raíz mayor } \\
\text { de Roy }\end{array}$ &, 462 & 9,244 & 2,000 & 40,000 &, 001 &, 316 &, 968 \\
\hline
\end{tabular}

Tabla 2. Medias obtenidas por el factor Estructura en las maquetas A, B y C.

\begin{tabular}{|c|c|c|c|c|}
\hline \multicolumn{5}{|c|}{ MEDIAS MARGINALES ESTIMADAS } \\
\hline \multirow{2}{*}{$\begin{array}{c}\text { Estructura } \\
\text { de la maqueta }\end{array}$} & Media & \multirow{2}{*}{ Error típico } & \multicolumn{2}{|c|}{ Intervalo de confianza 95\% } \\
\cline { 4 - 5 } & & & Límite inferior & Límite superior \\
\hline A & 1,952 &, 083 & 1,785 & 2,120 \\
\hline B & 2,291 &, 103 & 2,083 & 2,499 \\
\hline C & 2,122 &, 094 & 1,931 & 2,312 \\
\hline
\end{tabular}


Figura 3. Representación gráfica de las respectivas maquetas A, B y C.

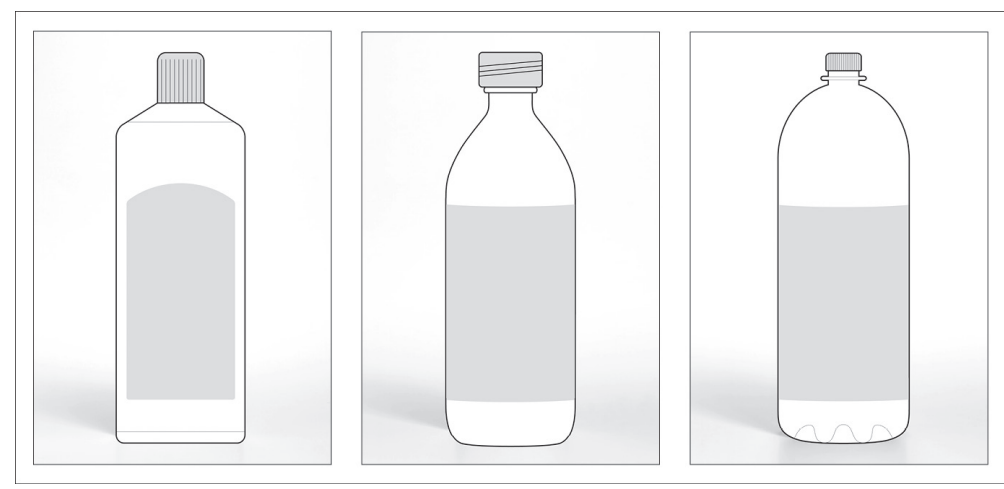

Fuente: Elaboración propia.

\subsection{Segunda hipótesis}

$\mathrm{H}_{2}$ El valor cromático base del envase es un componente del código visual.

Dado que el nivel de significación (sig.) relativo al factor Color_Base es 0,00 se puede rechazar la hipótesis nula y, en consecuencia, concluir que se detectan diferencias significativas entre los valores cromáticos base de las botellas (Tabla 3). Se puede aceptar la credibilidad de dicha hipótesis.

Asimismo es importante considerar la excelente potencia estadística del contraste $(1,00)$ la cual es superior a 0,80 .

Tabla 3. Valores estadísticos de significación y potencia de los contrastes relativos al factor Color_Base.

\begin{tabular}{|l|c|c|c|c|c|c|c|}
\hline \multicolumn{7}{|c|}{ COLOR_BASE } \\
\hline $\begin{array}{l}\text { Contrastes } \\
\text { multivariados }\end{array}$ & Valor & F & $\begin{array}{c}\text { GI de la } \\
\text { hipótesis }\end{array}$ & $\begin{array}{c}\text { GI del } \\
\text { error }\end{array}$ & Sig. & $\begin{array}{c}\text { Coeficiente } \\
\mathbf{r}^{2}\end{array}$ & $\begin{array}{c}\text { Potencia } \\
\text { observada }\end{array}$ \\
\hline Traza de Pillai &, 768 & 66,090 & 2,000 & 40,000 &, 000 &, 768 & 1,000 \\
\hline $\begin{array}{l}\text { Lambda } \\
\text { de Wilks }\end{array}$ &, 232 & 66,090 & 2,000 & 40,000 &, 000 &, 768 & 1,000 \\
\hline $\begin{array}{l}\text { Traza } \\
\text { de Hotelling }\end{array}$ & 3,304 & 66,090 & 2,000 & 40,000 &, 000 &, 768 & $\mathbf{1 , 0 0 0}$ \\
\hline $\begin{array}{l}\text { Raíz mayor } \\
\text { de Roy }\end{array}$ & 3,304 & 66,090 & 2,000 & 40,000 &, 000 &, 768 & $\mathbf{1 , 0 0 0}$ \\
\hline
\end{tabular}

Por otra parte, las medias marginales estimadas del factor Color_Base (Tabla 4) indican que la media más alta $(3,034)$ corresponde al valor cromático base 1 (muestra B1); en contraposición, los valores cromáticos base 2 (muestra B2) y 3 (muestra B3) han alcanzado unos resultados muy inferiores. 
Tabla 4. Medias marginales estimadas obtenidas por el factor Color_Base.

\begin{tabular}{|c|c|c|c|c|}
\hline \multicolumn{5}{|c|}{ MEDIAS MARGINALES ESTIMADAS } \\
\hline \multirow{2}{*}{ Color_Base } & Media & \multirow{2}{*}{ Error típico } & \multicolumn{2}{|c|}{ Intervalo de confianza 95\% } \\
\cline { 4 - 5 } & & & Límite inferior & Límite superior \\
\hline 1 & 3,034 &, 100 & 2,833 & 3,235 \\
\hline 2 & 1,608 &, 100 & 1,406 & 1,811 \\
\hline 3 & 1,722 &, 111 & 1,498 & 1,947 \\
\hline
\end{tabular}

\subsection{TERCERA HIPÓTESIS}

$\mathrm{H}_{3}$ El valor cromático auxiliar del envase es un componente del código visual.

Dado que el nivel de significación (sig.) relativo al factor Color_Auxiliar es 0,00 se puede rechazar la hipótesis nula y, en consecuencia, concluir que se detectan diferencias significativas entre los valores cromáticos auxiliares de los envases (Tabla 5). Se puede aceptar la credibilidad de dicha hipótesis.

Asimismo es importante considerar la excelente potencia estadística del contraste $(1,00)$ la cual es superior a 0,80 .

Tabla 5. Valores estadísticos de significación y potencia de los contrastes relativos al factor Color_Auxiliar.

\begin{tabular}{|l|c|c|c|c|c|c|c|}
\hline \multicolumn{7}{|c|}{ COLOR_AUXILIAR } \\
\hline $\begin{array}{l}\text { Contrastes } \\
\text { multivariados }\end{array}$ & Valor & F & $\begin{array}{c}\text { GI de la } \\
\text { hipótesis }\end{array}$ & $\begin{array}{c}\text { GI del } \\
\text { error }\end{array}$ & Sig. & $\begin{array}{c}\text { Coeficiente } \\
\mathbf{r}^{2}\end{array}$ & $\begin{array}{c}\text { Potencia } \\
\text { observada }\end{array}$ \\
\hline Traza de Pillai &, 551 & 24,544 & 2,000 & 40,000 &, 000 &, 551 & 1,000 \\
\hline $\begin{array}{l}\text { Lambda } \\
\text { de Wilks }\end{array}$ &, 449 & 24,544 & 2,000 & 40,000 &, 000 &, 551 & 1,000 \\
\hline $\begin{array}{l}\text { Traza } \\
\text { de Hotelling }\end{array}$ & 1,227 & 24,544 & 2,000 & 40,000 &, 000 &, 551 & 1,000 \\
\hline $\begin{array}{l}\text { Raíz mayor } \\
\text { de Roy }\end{array}$ & 1,227 & 24,544 & 2,000 & 40,000 &, 000 &, 551 & 1,000 \\
\hline
\end{tabular}

Por otra parte, las medias marginales estimadas del factor Color_Auxiliar (Tabla 6) indican que la media más alta $(2,688)$ corresponde al valor cromático auxiliar 1 (muestra A1); en contraposición, los valores cromáticos auxiliar 2 (muestra A2) y 3 (muestra A3) han alcanzado unos resultados muy inferiores. 
Tabla 6. Medias marginales estimadas obtenidas por el factor Color_Auxiliar.

\begin{tabular}{|c|c|c|c|c|}
\hline \multicolumn{5}{|c|}{ MEDIAS MARGINALES ESTIMADAS } \\
\hline \multirow{2}{*}{ Color_Auxiliar } & Media & \multirow{2}{*}{ Error típico } & \multicolumn{2}{|c|}{ Intervalo de confianza 95\% } \\
\cline { 4 - 5 } & & & Límite inferior & Límite superior \\
\hline $\mathbf{1}$ & $\mathbf{2 , 6 8 8}$ &, 117 & 2,451 & 2,924 \\
\hline $\mathbf{2}$ & 1,799 &, 093 & 1,612 & 1,986 \\
\hline $\mathbf{3}$ & 1,878 &, 122 & 1,633 & 2,124 \\
\hline
\end{tabular}

\subsection{CUARTA HIPÓTESIS}

A partir de este apartado se consideran algunos efectos de interacción específicos entre los factores que han intervenido en el experimento. En particular, se procede a analizar la cuarta hipótesis de trabajo la cual plantea los efectos de interacción producidos entre los valores cromáticos base y auxiliar. Dicha hipótesis se expone en los siguientes términos:

$\mathrm{H}_{4}$ Cuando se combina en un envase el valor cromático base con el valor cromático auxiliar se identifica la tipología de producto.

Dado que el nivel de significación (sig.) relativo a la interacción entre los factores Color_Base y Color_Auxiliar es 0,00 se puede rechazar la hipótesis nula y, en consecuencia, concluir que se detectan diferencias significativas entre los diferentes tratamientos experimentales (Tabla 7). En este sentido, dichas diferencias son debidas a las combinaciones que se establecen entre los valores cromáticos base y auxiliar. Se puede aceptar la credibilidad de dicha hipótesis.

Asimismo es importante considerar la excelente potencia estadística del contraste $(1,00)$ la cual es superior a 0,80 .

Tabla 7. Valores estadísticos de significación y potencia de los contrastes relativos a la interacción entre los factores Color_Base y Color_Auxiliar.

\begin{tabular}{|l|c|c|c|c|c|c|c|}
\hline \multicolumn{7}{|c|}{ COLOR_BASE + COLOR_AUXILIAR } \\
\hline $\begin{array}{l}\text { Contrastes } \\
\text { multivariados }\end{array}$ & Valor & $\mathbf{F}$ & $\begin{array}{c}\text { GI de la } \\
\text { hipótesis }\end{array}$ & GI del error & Sig. & $\begin{array}{c}\text { Coeficiente } \\
\mathbf{r}^{2}\end{array}$ & $\begin{array}{c}\text { Potencia } \\
\text { observada }\end{array}$ \\
\hline Traza de Pillai &, 627 & 15,981 & 4,000 & 38,000 &, 000 &, 627 & $\mathbf{1 , 0 0 0}$ \\
\hline $\begin{array}{l}\text { Lambda } \\
\text { de Wilks }\end{array}$ &, 373 & 15,981 & 4,000 & 38,000 &, 000 &, 627 & $\mathbf{1 , 0 0 0}$ \\
\hline $\begin{array}{l}\text { Traza } \\
\text { de Hotelling }\end{array}$ & 1,682 & 15,981 & 4,000 & 38,000 &, 000 &, 627 & $\mathbf{1 , 0 0 0}$ \\
\hline $\begin{array}{l}\text { Raíz mayor } \\
\text { de Roy }\end{array}$ & 1,682 & 15,981 & 4,000 & 38,000 &, 000 &, 627 & $\mathbf{1 , 0 0 0}$ \\
\hline
\end{tabular}


Por otra parte, las medias marginales estimadas de la interacción factorial Color_Base y Color_Auxiliar indican que la media más alta $(4,317)$ corresponde a la combinación entre el valor cromático base 1 y el auxiliar 1 (Tabla 8). Las restantes medias han presentado unos valores muy inferiores. En consecuencia, dicha formulación cromática se manifiesta como indicativa de la tipología de producto: leche entera.

Tabla 8. Conjunto de medias obtenidas por la interacción factorial entre Color_Base y Color_Auxiliar.

\begin{tabular}{|c|c|c|c|c|c|}
\hline \multicolumn{6}{|c|}{ MEDIAS MARGINALES ESTIMADAS } \\
\hline \multirow{2}{*}{ Color_Base } & \multirow{2}{*}{ Color_Auxiliar } & \multirow{2}{*}{ Media } & \multirow{2}{*}{ Error típico } & \multicolumn{2}{|c|}{ Intervalo de confianza al $95 \%$} \\
\hline & & & & Límite inferior & Límite superior \\
\hline \multirow{3}{*}{1} & 1 & 4,317 & ,090 & 4,128 & 4,507 \\
\hline & 2 & 2,325 & ,172 & 1,978 & 2,673 \\
\hline & 3 & 2,460 & ,179 & 2,098 & 2,822 \\
\hline \multirow{3}{*}{2} & 1 & 1,833 & , 180 & 1,469 & 2,198 \\
\hline & 2 & 1,476 & ,090 & 1,295 & 1,657 \\
\hline & 3 & 1,516 &, 116 & 1,282 & 1,750 \\
\hline \multirow{3}{*}{3} & 1 & 1,913 & ,170 & 1,569 & 2,257 \\
\hline & 2 & 1,595 & ,093 & 1,408 & 1,782 \\
\hline & 3 & 1,659 & 133 & 1,391 & 1,927 \\
\hline
\end{tabular}

\subsection{Quinta hipótesis}

En último lugar, se procede a analizar la quinta hipótesis de trabajo la cual plantea los efectos de interacción producidos entre la estructura del envase y los valores cromáticos base y auxiliar. Dicha hipótesis se expone en los siguientes términos:

$\mathrm{H}_{5}$ Cuando se combina la estructura del envase con los valores cromáticos base y auxiliar se identifica la tipología de producto.

Dado que el nivel de significación ( $\mathrm{sig}$.) relativo a la interacción entre los factores Estructura,CColor_Base y Color_Auxiliar es 0,244 se tiene que aceptar la hipótesis nula y, en consecuencia, concluir que no se detectan diferencias estadísticamente significativas entre los diferentes tratamientos experimentales (Tabla 9). En este sentido, no se manifiestan diferencias lo suficientemente relevantes cuando intervienen 
conjuntamente las tres variables independientes. Por tanto, no se puede aceptar la credibilidad de dicha hipótesis.

Asimismo es importante considerar la baja potencia estadística del contraste $(0,526)$ la cual es muy inferior a 0,80 . En esta circunstancia, la probabilidad de cometer un error Tipo II es muy alta.

Por otra parte, las medias marginales estimadas de la interacción factorial Estructura, Color_Base y Color_Auxiliar (Tabla 10) indican que la media más alta $(4,524)$ corresponde a la combinación entre la estructura de la maqueta $\mathrm{B}$, el valor cromático base 1 (blanco) y el auxiliar 1 (azul oscuro). A pesar de ello, no se observan diferencias importantes respecto de las maquetas A y $\mathrm{C}$ dado que las medias en ambos casos son sólo ligeramente inferiores (4,167 y 4,262 respectivamente).

De acuerdo con estos resultados, se pone de manifiesto que la influencia del factor Estructura no ha sido lo suficientemente relevante en la constitución del código visual. Aunque las medias obtenidas para los tratamientos visuales MAB1A1 (Figura 4), MBB1A1 (Figura 5) y MCB1A1 (Figura 6) sean altas, los valores son muy similares entre sí y, en consecuencia, no se establece una diferencia estadísticamente evidente. Es por esta razón -la igualdad estadística de las medias- que no se puede rechazar, en este caso, la hipótesis nula. Sin embargo, sí que se ha encontrado suficiente evidencia en el hecho de que el código visual se circunscriba al ámbito cromático.

Tabla 9. Valores estadísticos de significación y potencia de los contrastes relativos a la interacción entre los factores Estructura, Color_Base y Color_Auxiliar.

\begin{tabular}{|l|c|c|c|c|c|c|c|}
\hline \multicolumn{7}{|c|}{ ESTRUCTURA + COLOR_BASE + COLOR_AUXILIAR } \\
\hline $\begin{array}{l}\text { Contrastes } \\
\text { multivariados }\end{array}$ & Valor & $\mathbf{F}$ & $\begin{array}{c}\text { GI de la } \\
\text { hipótesis }\end{array}$ & $\begin{array}{c}\text { GI del } \\
\text { error }\end{array}$ & Sig. & $\begin{array}{c}\text { Coeficiente } \\
\mathbf{r}^{2}\end{array}$ & $\begin{array}{c}\text { Potencia } \\
\text { observada }\end{array}$ \\
\hline Traza de Pillai &, 244 & 1,371 & 8,000 & 34,000 &, 244 &, 244 &, $\mathbf{5 2 6}$ \\
\hline $\begin{array}{l}\text { Lambda } \\
\text { de Wilks }\end{array}$ &, 756 & 1,371 & 8,000 & 34,000 &, $\mathbf{2 4 4}$ &, 244 &, $\mathbf{5 2 6}$ \\
\hline $\begin{array}{l}\text { Traza } \\
\text { de Hotelling }\end{array}$ &, 323 & 1,371 & 8,000 & 34,000 &, $\mathbf{2 4 4}$ &, 244 &, $\mathbf{5 2 6}$ \\
\hline $\begin{array}{l}\text { Raíz mayor } \\
\text { de Roy }\end{array}$ &, 323 & 1,371 & 8,000 & 34,000 &, $\mathbf{2 4 4}$ &, 244 &, $\mathbf{5 2 6}$ \\
\hline
\end{tabular}

Figura 4: MAB1A1

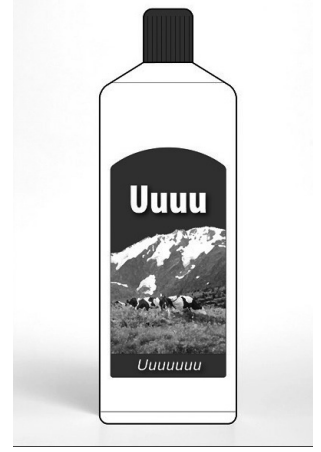

Figura 5: MBB1A1

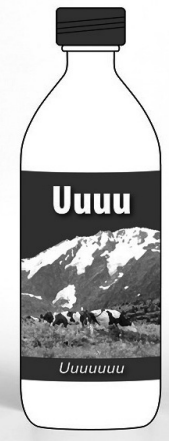

Figura 6: MCB1A1

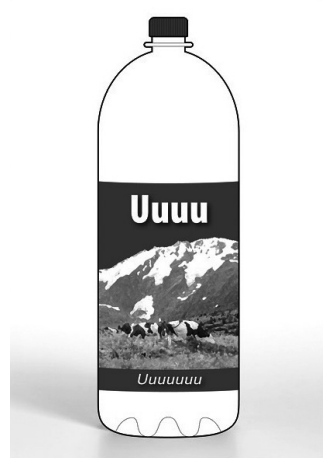


Tabla 10. Medias obtenidas por la interacción factorial entre Estructura, Color_Base y Color_Auxiliar.

\begin{tabular}{|c|c|c|c|c|c|c|}
\hline \multicolumn{7}{|c|}{ MEDIAS MARGINALES ESTIMADAS } \\
\hline \multirow{2}{*}{$\begin{array}{c}\text { Estructura } \\
\text { de la } \\
\text { maqueta }\end{array}$} & \multirow{2}{*}{$\begin{array}{c}\text { Color_ } \\
\text { Base }\end{array}$} & \multirow{2}{*}{$\begin{array}{l}\text { Color } \\
\text { Auxiliar }\end{array}$} & \multirow{2}{*}{ Media } & \multirow{2}{*}{$\begin{array}{l}\text { Error } \\
\text { típico }\end{array}$} & \multicolumn{2}{|c|}{$\begin{array}{c}\text { Intervalo de confianza } \\
\text { al } 95 \% \\
\end{array}$} \\
\hline & & & & & Límite inferior & Límite superior \\
\hline \multirow{9}{*}{ A } & \multirow{3}{*}{1} & 1 & 4,167 & ,136 & 3,892 & 4,441 \\
\hline & & 2 & 1,976 & ,182 & 1,609 & 2,344 \\
\hline & & 3 & 1,976 & , 175 & 1,622 & 2,330 \\
\hline & \multirow{3}{*}{2} & 1 & 1,714 & , 191 & 1,329 & 2,099 \\
\hline & & 2 & 1,452 & ,114 & 1,222 & 1,683 \\
\hline & & 3 & 1,357 & , 127 & 1,101 & 1,613 \\
\hline & \multirow{3}{*}{3} & 1 & 1,810 & ,184 & 1,437 & 2,182 \\
\hline & & 2 & 1,476 & 109 & 1,256 & 1,696 \\
\hline & & 3 & 1,643 & ,159 & 1,321 & 1,964 \\
\hline \multirow{9}{*}{ B } & \multirow{3}{*}{1} & 1 & 4,524 & ,133 & 4,255 & 4,792 \\
\hline & & 2 & 2,429 & ,196 & 2,032 & 2,825 \\
\hline & & 3 & 2,857 & ,222 & 2,408 & 3,306 \\
\hline & \multirow{3}{*}{2} & 1 & 2,000 & ,204 & 1,587 & 2,413 \\
\hline & & 2 & 1,500 & ,124 & 1,249 & 1,751 \\
\hline & & 3 & 1,738 & ,149 & 1,438 & 2,039 \\
\hline & \multirow{3}{*}{3} & 1 & 2,190 & ,221 & 1,743 & 2,638 \\
\hline & & 2 & 1,619 & ,136 & 1,344 & 1,894 \\
\hline & & 3 & 1,762 & 180 & 1,399 & 2,125 \\
\hline \multirow{9}{*}{ C } & \multirow{3}{*}{1} & 1 & 4,262 & ,156 & 3,946 & 4,578 \\
\hline & & 2 & 2,571 & ,224 & 2,119 & 3,023 \\
\hline & & 3 & 2,548 & ,216 & 2,111 & 2,984 \\
\hline & \multirow{3}{*}{2} & 1 & 1,786 & ,194 & 1,393 & 2,178 \\
\hline & & 2 & 1,476 & ,119 & 1,235 & 1,717 \\
\hline & & 3 & 1,452 & ,128 & 1,193 & 1,712 \\
\hline & \multirow{3}{*}{3} & 1 & 1,738 & 187 & 1,361 & 2,115 \\
\hline & & 2 & 1,690 & 158 & 1,371 & 2,010 \\
\hline & & 3 & 1,571 & 149 & 1,270 & 1,873 \\
\hline
\end{tabular}

\section{Conclusiones}

De acuerdo con los datos obtenidos, se han detectado diferencias estadísticamente significativas que ponen de manifiesto que las variables Estructura, Color_Base y Color_Auxiliar actúan, en principio, como componentes activos del código visual. 
En primer lugar, se ha podido constatar -según los efectos principales analizados- que los contrastes estadísticos confirman que entre los tratamientos visuales se han observado diferencias relevantes en relación a la estructura del envase. En este sentido, la maqueta B ha sido la opción preferida para identificar la tipología de producto (leche).

En cuanto a la variable Color_Base, los contrastes estadísticos confirman que entre los valores cromáticos utilizados se establecen diferencias significativas. En este caso, la diferencia entre medias muestra que el valor cromático base 1 -cuando la botella de leche es blanca- es el que mejor congenia con dicha tipología de producto.

Por otra parte, los contrastes estadísticos relativos a la variable Color_Auxiliar indican también que entre dichos valores cromáticos se evidencian diferencias significativas. En este punto, se pone de manifiesto que el valor cromático auxiliar permite identificar la tipología de leche. Es decir, según los resultados obtenidos, los sujetos experimentales asocian el valor cromático auxiliar 1 (azul) con la leche entera.

En segundo lugar, se ha podido constatar -según los efectos de interacción factorial- que los contrastes estadísticos entre Color_Base y Color_Auxiliar ponen de manifiesto una diferencia significativa de medias entre los tratamientos experimentales. Dicha diferencia emerge cuando se combina siempre el valor cromático base 1 (blanco) con el valor cromático auxilar 1 (azul). Esto ha permitido inferir que existe un código cromático específico para identificar la tipología de producto: leche entera.

Con respecto a la interacción factorial Estructura,CColor_Base y Color_Auxiliar, los contrastes estadísticos no consiguen valores de significación aceptables. Es decir, la intervención de la variable Estructura no ha tenido efectos relevantes sobre los sujetos experimentales y, por lo tanto, su influencia como componente activo del código visual no se ha podido constatar.

Los resultados de las pruebas de contraste indican que el código es fundamentalmente cromático, dado que la intervención de las variables Color_Base y Color_Auxiliar relega o anula el hipotético efecto provocado por la estructura del envase. Parece, por tanto, que el efecto principal del factor Estructura pierde potencia comunicativa cuando interactúa con los restantes factores.

En este caso, se pueden contemplar tres posibles explicaciones. Primera, que los niveles del factor Estructura no hayan tenido un mayor contraste visual que permita la fácil diferenciación de las maquetas. Segunda, que no exista una estructura de envase prototípica para determinar la identidad tipológica del producto. Tercera, considerar que la participación de una mayor muestra de sujetos experimentales hubiera podido incrementar el nivel de significación del contraste no superado.

Para terminar, a partir de este trabajo se abren nuevas líneas de investigación que permitirán, por una parte, estudiar los umbrales perceptivos en relación a las estructuras de los envases pero, al mismo tiempo, analizar el código cromático de los productos o, incluso, la forma tipográfica como probable componente del código visual. 


\section{Referencias bibliográficas}

AAKER, David; MYERS, John (1984). Management de la publicidad. Perspectivas prácticas. Barcelona: Editorial Hispano Europea.

ALBA, Joseph W.; HUTCHINSON, J. Wesley (1987). Dimensions of consumer expertise. En: Journal of Consumer Research, vol. 13, pp. 411-453.

AMBROSE, Gavin; HARRIS, Paul (2011). Packaging de la marca. Barcelona: Parramón Ediciones.

ARNHEIM, Rudolf (2005). Arte y percepción visual. Madrid: Alianza Editorial.

BASSAT, Luís (1994). El libro rojo de la publicidad. Barcelona: Ediciones Folio.

BASU, Kunal (1993). Consumer's categorization processes: an examination with two alternative methodological paradigms. En: Journal of Consumer Psychology, vol. 2, pp. 97-121.

BERKOWITZ, Marvin (1987). Product shape as a design innovation strategy. En: The Journal of Product Innovation Management, núm. 4, pp. 274-283.

BERTALANFFY, Ludwing von (1981). Teoría general de los sistemas. Fundamentos, desarrollo, aplicaciones. Madrid: Fondo de Cultura Económica.

BLOCH, Peter H. (1995). Seeking the ideal form: product design and consumer response. En: Journal of Marketing, vol. 59, Issue. 3, pp. 16-29.

BUNGE, Mario (1989). La investigación científica. Barcelona: Ariel.

CALVER, Giles (2004). ¿Qué es el packaging? Barcelona: Gustavo Gili.

CAPELLA, Juli; ÚBEDA, Ramón (2003). Cocos, copias y coincidencias. Barcelona: Electa.

CERVERA, Ángel Luis (2003). Envase y embalaje, la venta silenciosa. Madrid: ESIC.

COLET, Jordi (2012). La identidad visual de los envases. Una aproximación a la función publicitaria del packaging. [Tesis doctoral en línea] Bellaterra: Universidad Autónoma de Barcelona. Disponible en http://www.tdx.cat/handle/10803/145037

CONNOLLY, A.; DAVIDSON, L. (1996). How does design affect decisions at point of sale? En: Journal of Brand Management, vol. 4, núm. 2, pp.100-107.

COSTA, Joan (2004). La imagen de marca. Barcelona: Ediciones Paidós.

CREUSEN, Mariëlle; SCHOORMANS, Jan (2005). The different roles of product appearance in consumer choice. En: The Journal of Product Innovation Management, vol. 22, pp. 63-81.

DEMBER, W. (1990). Psicologia de la percepción. Madrid: Alianza Editorial.

DONDIS, Donis (2004). La sintaxis de la imagen. Barcelona: Editorial Gustavo Gili.

DEFLEUR, Melvin; BALL-ROKEACH, Sandra (1982). Teorías de la comunicación de masas. Barcelona: Editorial Paidós.

ECO, Umberto (1972). La estructura ausente. Barcelona: Editorial Lumen.

- - (1977). Tratado de semiótica general. Barcelona: Editorial Lumen.

FESTINGER, Leon (1975). Teoría de la disonancia cognitiva. Madrid: Centro de Estudios Políticos y Constitucionales.

GARBER, Lawrence (1995). The package appearance in choice. En: Advances in Consumer Research, vol. 22, pp. 653-660.

HELLER, Eva (2005). Psicología del color. Barcelona: Gustavo Gili. 
HOYER, Wayne (1984). An examination of consumer decision making for a common repeat purchase product. En: Journal of Consumer Research, vol. 11, pp. 822-829.

IGARTUA, Juan José (2006). Métodos cuantitativos de investigación en comunicación. Barcelona: Editorial Bosch.

LÁZARO, Patricia et al. (2007). La recepción, un límite a la creación. En: Trípodos. Barcelona: Servicio de Diseño y Publicaciones Blanquerna. Número extraordinario, pp. 685-704.

LOKEN, Barbara; WARD, James (1990). Alternative approaches to understanding the determinants of tipicality. En: Journal of Consumer Research, 1990, vol. 17, pp. 111-126.

MAEDA, John (2010). Las leyes de la simplicidad. Barcelona: Gedisa.

MARTÍNEZ, José Manuel (2010). La influencia del Aspecto en la eficiencia del mensaje gráfico publicitario. [Tesis doctoral en línea] Bellaterra: Universidad Autónoma de Barcelona. Disponible en http://hdl.handle.net/10803/4161

MOLES, Abraham (1976). Teoría de la información y percepción estética. Madrid: Ediciones Júcar, 1976.

MORGADO, Ignacio (2012). Cómo percibimos el mundo. Una exploración de la mente y los sentidos. Barcelona: Editorial Ariel.

PHILIPS, H.; BRADSHAW, R. (1993). How customers actually shop: customer interaction with the point of sale. En: Journal of the Market Research Society, vol. 35, núm. 1, pp. 51-62.

RETTIE, Ruth; BREWER, Carol (2000). The verbal and visual components of package design. En: Journal of Product \& Brand Management, vol. 9, núm. 1, pp. 56-70.

ROM, Josep (2002). Els fonaments del disseny gràfic. Barcelona: Trípodos.

ROSCH, Eleanor; MERVIS, Carolyn (1975). Family resemblances: studies in the internal structure of categories. En: Cognitive Psychology, vol. 7, pp. 573-605.

SAPERAS, Enric (1985). La sociología de la comunicación de masas en los Estados Unidos. Barcelona: Editorial Ariel.

SCHNAKE, Hugo (1990). El comportamiento del consumidor. México: Editorial Trillas.

SCHOORMANS, Jan; ROBBEN, Henry (1997). The effect of new package design on product attention, categorization and evaluation. En: Journal of Economic Psychology, vol. 18, pp. 271-287.

SIERRA, Benjamín; FALCES, Carlos (2001). El papel de la apariencia visual del diseño de los envases en el reconocimiento del producto, de la variedad y de la marca. En: RUIZ, Salvador; ALONSO, Javier. Experiencias y casos de comportamiento del consumidor. Madrid: ESIC, pp. 131-143.

STEWART, Bill (2008). Packaging. Manual de diseño y producción. Barcelona: Gustavo Gili.

TENA, Daniel (1998). La influencia de la composición gráfica en la elección de un bloque de texto escrito. [Tesis doctoral en línea] Bellaterra: Universidad Autónoma de Barcelona. Disponible en http://www.tdx.cat/handle/10803/4135

VERYZER, Robert (1995). The place of product design and aesthetics in consumer research. En: Advances in Consumer Research, vol. 22, pp. 641-645. 
VIDALES, María Dolores (2003). El mundo del envase. Manual para el diseño y producción de envases y embalajes. Barcelona: Editorial Gustavo Gili.

VIGLIETTI, M. (1975). La psicología de la forma y la Gestalt Theorie. Barcelona: Ediciones Don Bosco.

VIÑOLAS, Joaquim (2005). Diseño ecológico. Barcelona: Blume.

WARD, James; LOKEN, Barbara (1988). The generality of typicality effects on preference and comparison: an exploratory test. En: Advances in Consumer Research, vol. 15, pp. 55-61. 